\title{
The impact of social distancing on pediatric neurosurgical emergency referrals during the COVID-19 pandemic: a prospective observational cohort study
}

\author{
Edward W Dyson $^{1}$ (1) $\cdot$ Claudia L Craven $^{1} \cdot$ Martin M Tisdall ${ }^{1,2} \cdot$ Gregory A James $^{1,2}$
}

Received: 23 June 2020 / Accepted: 25 June 2020 / Published online: 3 July 2020

(C) Springer-Verlag GmbH Germany, part of Springer Nature 2020

\section{Dear Editor:}

During the 2020 coronavirus pandemic, most countries in the world have employed social distancing measures to limit the spread of the novel coronavirus SARS-CoV-2 [1]. In their most extreme form, these are colloquially known as "lockdown" and include the prohibition of all but essential travel outside the home and closure of schools.

There has been widespread concern amongst the pediatric medical community that these measures might discourage parents from presenting their children to hospital in the event of significant illness. In addition, there has been considerable media speculation that social distancing measures might result in an increase in domestic abuse (in children referred to as non-accidental injury (NAI)) [2].

A considerable proportion of the workload of a pediatric neurosurgery department consists of acute referrals from emergency departments to the "on-call" resident neurosurgeon. We sought to investigate whether a change in referral volume and patterns was observed during the period of socalled lockdown in the UK, with a focus on the pattern of trauma referrals.

We carried out a single-centre prospective observational cohort study of all referrals to a regional pediatric neurosurgery unit during the first 6 weeks of the period of extreme social distancing measures in place in the UK during the coronavirus pandemic and compared this to a retrospectively collected, contemporary, seasonally matched control period of the same duration.

Edward W Dyson

edwarddyson@nhs.net

1 Department of Neurosurgery, Great Ormond Street Hospital for Children NHS Foundation Trust, London, UK

2 UCL Institute of Child Health, London, UK
Inclusion criteria All patients referred to the acute pediatric neurosurgery service between the 23rd of March 2020 and 3rd of May 2020 ("during COVID-19") and between the 25th of March 2019 and 5th of May 2019 ("pre-COVID19"). Telephone queries about post-operative patients were excluded in order to focus on new presentations only.

Baseline demographic data were obtained from the prospectively maintained electronic patient record system. Patients were grouped by referral pathology. Subgroup analysis of trauma referrals was carried out including trauma mechanism and imaging findings. Since our a priori hypothesis was an increase in trauma referrals, we grouped together non-trauma referrals for the purpose of statistical analysis.

Unpaired $t$ test was used to compare the mean age in the two comparison groups. The chi-squared test was used to compare proportions of referrals. All statistical tests were performed on Prism 8.0c (GraphPad Software Inc., CA).

The study was approved as an audit by the local clinical governance department.

In the pre-COVID-19 group, 68 patients (37 M: $31 \mathrm{~F})$ aged $5.63 \pm 5.66$ years (mean $\pm \mathrm{SD}$ ) were referred. In the during COVID-19 group, 78 patients ( $45 \mathrm{M}: 33 \mathrm{~F}$ ) aged $4.84 \pm 4.91$ were referred. There was no significance in the gender $(p=0.69)$ or age $(p=0.37)$ of patients referred between the two groups.

Figure 1 summarises the acute referral pathologies between the two groups. Most acute referrals were trauma-related. There was a non-significant increase in the number of trauma referrals between the cohorts (17 and 27 patients respectively, $p=0.35$ ).

Figure 2 summarises the mechanisms of injury encountered and the associated imaging findings. There was no significant difference in any of the trauma mechanisms between the two cohorts. There was a significant increase in the number of trauma patients with intracranial haemorrhagic findings on imaging; however, no significant increase in any one individual subgroup. 


\begin{tabular}{|c|c|c|c|c|}
\hline & $\begin{array}{l}\text { Pre-Covid-19 } \\
(\mathrm{n}=68)\end{array}$ & $\begin{array}{l}\text { During Covid-19 } \\
(\mathrm{n}=78)\end{array}$ & P-value & Absolute Change in Referrals from Pre-Covid-19 to During Covid-19 \\
\hline 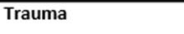 & 17 & 27 & 0.35 & \\
\hline Non-trauma & 51 & 51 & 0.60 & \\
\hline Hydrocephalus & 28 & 25 & 0.44 & \\
\hline Infection & 6 & 4 & 0.41 & \\
\hline Congenital & 4 & 6 & 0.69 & \\
\hline Oncology & 5 & 7 & 0.74 & \\
\hline Spine (non-trauma) & 5 & 2 & 0.20 & \\
\hline Neurovascular & 1 & 2 & 0.65 & \\
\hline Non-neurosurgical & 2 & 5 & 0.35 & \\
\hline & & & & $\begin{array}{l}1 \\
5\end{array}$ \\
\hline
\end{tabular}

Fig. 1 Summary of referral pathology and absolute change

Overall, our data shows a small increase in the number of emergency pediatric neurosurgical referrals during the COVID-19 lockdown in the UK. A particular increase in trauma referrals was observed, although this failed to reach statistical significance, with a stable number of non-trauma referrals. One would not expect a change in human behaviour to have any impact on the true incidence of the so-called spontaneous pathology in the population; however, the proportion which presents to hospital may be affected. These data do not therefore support concerns that serious, time-critical intracranial pathology such as brain tumours and hydrocephalus are being inappropriately kept away from hospital as a result of social distancing.

Social distancing has resulted in a reduction in emergency department attendances overall [3]. Our data demonstrates that this has not been accompanied by a reduction in acute neurosurgical presentations. This has important implications in planning service delivery for any future similar episodes. Whilst we did observe a trend towards more NAI cases, this was proportionate to the overall increase in trauma referrals. No individual sub-group of trauma referrals demonstrated a significant increase. An increase in any of these groups in the community cannot be excluded, and indeed is possible given that our data looks solely at inter-hospital referrals.

We also observed a significant increase in the incidence of traumatic intracranial haemorrhage amongst trauma patients referred during the lockdown period. Most children referred with head trauma had haemorrhagic findings on CT scan, compared with a small minority in the pre-COVID-19 group. This raises the concern that the severity of neurological trauma sustained during the lockdown may have been greater. Our data cannot exclude an increase in NAI which does not present to hospital and this is worthy of further research.

Acknowledgements We are grateful for the assistance of Kristian Aquilina, Owase Jeelani, Zubair Tahir, and Dominic Thompson, Consultant Neurosurgeons, Great Ormond Street Hospital for Children NHS Foundation Trust, London, UK, for their assistance in gathering the data.

\begin{tabular}{|c|c|c|c|c|}
\hline & $\begin{array}{l}\text { Pre-Covid-19 } \\
\text { (n=17) }\end{array}$ & $\begin{array}{l}\text { During Covid-19 } \\
(\mathrm{n}=27)\end{array}$ & P-value & Absolute Cnange \\
\hline \multicolumn{5}{|l|}{ Mechanism } \\
\hline Suspected NAI & 2 & 6 & 0.46 & 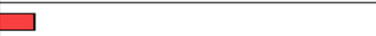 \\
\hline Fall from height & 11 & 10 & 0.30 & \\
\hline Fall from standing & 2 & 2 & \begin{tabular}{|l|}
0.70 \\
\end{tabular} & \\
\hline Birth Trauma & 1 & 3 & 0.60 & $\square$ \\
\hline Bicycle or Scooter & \begin{tabular}{|l|}
1 \\
\end{tabular} & 4 & 0.41 & \\
\hline Trampoline & 0 & 2 & 0.27 & $\square$ \\
\hline \multicolumn{5}{|l|}{ Imaging Findings } \\
\hline Intracranial Haemorrhage & 4 & 22 & $0.04^{*}$ & * \\
\hline Contusion & 0 & 5 & 0.09 & \\
\hline Traumatic SAH & 2 & 3 & 0.95 & 曰 \\
\hline $\mathrm{SDH}$ & 1 & 9 & 0.08 & \\
\hline $\mathrm{EDH}$ & 1 & 5 & 0.29 & \\
\hline Skull fracture & 13 & 18 & 0.81 & \\
\hline Spinal Injury & \begin{tabular}{|l|l|}
0 \\
\end{tabular} & 1 & 0.43 & $\square$ \\
\hline
\end{tabular}

Fig. 2 Summary of trauma mechanism and imaging findings and absolute change 
Code availability Not applicable.

Authors' contributions EWD and CLC collected the data. EWD wrote the manuscript. All authors reviewed the manuscript.

Data availability Anonymised data supporting the findings of this study are available from the corresponding author, EWD, upon reasonable request.

\section{Compliance with ethical standards}

Conflict of interest The authors have no personal, financial, or institutional interest in any of the drugs, materials, or devices described in this article.

Ethics approval This project was registered with the Great Ormond Street Hospital for Children NHS Foundation Trust Clinical Governance Department.

Consent to participate Not applicable.
Consent for publication Not applicable. N

\section{References}

1. Remuzzi A, Remuzzi G (2020) COVID-19 and Italy: what next? Lancet 395:1225-1228. https://doi.org/10.1016/S01406736(20)30627-9

2. Mulpuri K, Slobogean BL, Tredwell SJ (2011) The epidemiology of nonaccidental trauma in children. Clin Orthop Relat Res 469:759 767. https://doi.org/10.1007/s11999-010-1565-4

3. Lazzerini M, Barbi E, Apicella A, Marchetti F, Cardinale F, Trobia G (2020) Delayed access or provision of care in Italy resulting from fear of COVID-19. Lancet Child Adolesc Health 4:e10-e11. https:// doi.org/10.1016/S2352-4642(20)30108-5

Publisher's note Springer Nature remains neutral with regard to jurisdictional claims in published maps and institutional affiliations. 